\title{
Iterative Homotopy Harmonic Balance Approach for Determining the Periodic Solution of a Strongly Nonlinear Oscillator
}

\author{
Huaxiong Chen ${ }^{1}$ and Mingkang $\mathrm{Ni}^{1,2}$ \\ ${ }^{1}$ Department of Mathematics, East China Normal University, Shanghai 200241, China \\ ${ }^{2}$ Department of Mathematics, Shanghai Key Laboratory of PMMP, East China Normal University, Shanghai 200241, China \\ Correspondence should be addressed to Huaxiong Chen; chxecnu@163.com
}

Received 9 August 2015; Accepted 15 October 2015

Academic Editor: Tito Busani

Copyright (C) 2015 H. Chen and M. Ni. This is an open access article distributed under the Creative Commons Attribution License, which permits unrestricted use, distribution, and reproduction in any medium, provided the original work is properly cited.

A novel approach about iterative homotopy harmonic balancing is presented to determine the periodic solution for a strongly nonlinear oscillator. This approach does not depend upon the small/large parameter assumption and incorporates the salient features of both methods of the parameter-expansion and the harmonic balance. Importantly, in obtaining the higher-order analytical approximation, all the residual errors are considered in the process of every order approximation to improve the accuracy. With this procedure, the higher-order approximate frequency and corresponding periodic solution can be obtained easily. Comparison of the obtained results with those of the exact solutions shows the high accuracy, simplicity, and efficiency of the approach. The approach can be extended to other nonlinear oscillators in engineering and physics.

\section{Introduction}

Considerable attention has been paid to the study of nonlinear problems not only in all areas of engineering but also in physics and other disciplines, since world is nonlinear essentially and most phenomena are modeled by nonlinear differential equations. However, in general, it is very difficult to get exact solutions for those problems. Thus, to find approximate analytic solutions to these nonlinear problems has been the desire of many researchers for a long time.

During the past few decades, there are many analytical methods used to find approximate solutions of various types of nonlinear equations, such as the variational iteration method [1-4], He's parameter-expansion method [57], harmonic balance method [8-12], homotopy perturbation method [13-17], energy balance method [18, 19], and deltaperturbation [20].

Among these analytical methods, the iterative homotopy harmonic balancing [21] (denoted as IHHB for abbreviation) is a novel and effective technique for solving some strongly nonlinear oscillators. This approach incorporates the salient features of both methods of the parameter-expansion and the harmonic balance. By constructing a parameter $p$ which is considered as a "small parameter" in the harmonic balance process, the IHHB does not depend upon the large/small parameter assumption and can get high-order analytical approximations easily. Hence, the application of IHHB can be found in various nonlinear problems [21-23].

In this paper, we consider the following strongly nonlinear oscillator with nonpolynomial term [6]

$$
\begin{aligned}
\ddot{y}+\alpha y+\beta y^{3}+\gamma y^{1 / 3} & =0, \\
y(0) & =A, \\
y^{\prime}(0) & =0,
\end{aligned}
$$

where the over-dot denotes the derivative with respect to the time $t$ and $A$ is the amplitude of the oscillation.

When $\alpha=\beta=0, \gamma=1$, (1) becomes a truly nonlinear oscillator introduced by Mickens [12] and has been studied by many other investigators [11, 16, 17]. When $\alpha=1, \beta=\epsilon$, $\gamma=0$, (1) reduces to a well-known oscillator namely Duffing equation. By simple analysis [6], we know that (1) has periodic solution when $\alpha+\beta A^{2}+\gamma A^{-2 / 3}>0$. 
To get higher-order analytical and accurate approximations for the frequency and solution of this oscillator, IHHB solving is set up. More importantly, in obtaining the higherorder analytical approximation, all the residual errors are considered in the process of every order approximation to improve the accuracy. Excellent agreement of the approximate solution and frequency with the exact ones has been demonstrated and discussed. As can be seen, the results presented in this paper reveal that IHHB is effective and convenient for some nonlinear oscillators with nonpolynomial terms.

\section{Basic Ideas of Iterative Homotopy Harmonic Balancing Approach}

Consider the following second-order systems:

$$
\begin{aligned}
\ddot{y}-f(y) & =0, \\
y(0) & =A, \\
\dot{y}(0) & =0,
\end{aligned}
$$

where the over-dot denotes the derivative with respect to the time $t$ and $A$ is the amplitude of the oscillations. For convenience, we assume $f(-y)=-f(y)$.

By introducing a new independent variable $\tau=\omega t$, then (2) becomes

$$
\begin{aligned}
\omega^{2} y^{\prime \prime}-f(y) & =0, \\
y(0) & =A, \\
y^{\prime}(0) & =0,
\end{aligned}
$$

where prime denotes the derivative with respect to $\tau$ and $\omega$ is an angular frequency to be determined.

Let the periodic solution $y(\tau)$ of (3) exist and assume that it can be expressed by such a set of base functions

$$
\{\cos (2 m-1) \tau \mid m=1,2,3, \ldots\} .
$$

According to (4), the initial approximate periodic solution satisfying initial conditions should be

$$
y_{0}(\tau)=A \cos \tau, \quad \tau=\omega_{0} t,
$$

where $\omega_{0}$ is an unknown constant to be determined later.

Here, we will use the residual to improve the accuracy. Substituting (5) into (3), the initial residual can be obtained:

$$
R_{0}(\tau)=\omega_{0}^{2} y_{0}^{\prime \prime}-f\left(y_{0}\right) .
$$

If $R_{0}(\tau)=0$, then $y_{0}(\tau)$ happens to be the exact solution. However, in general, such case will not appear for nonlinear problems.

According to harmonic balance method, no secular term in (6) requires eliminating contributions proportional to $\cos \tau$, through which the unknown constant $\omega_{0}$ can be determined. Then, the zero-order approximation solution is obtained of the form

$$
y_{0}(\tau)=A \cos \tau, \quad \tau=\omega_{0} t
$$

with initial residual

$$
R_{0}(\tau)=\omega_{0}^{2} y_{0}^{\prime \prime}-f\left(y_{0}\right)
$$

In the following, we introduce a bookkeeping parameter $p$ with values in the interval $[0,1]$, denote the $y(\tau), \omega$ as $y(\tau, p), \omega(p)$, and then expand $y(\tau, p)$ and $\omega^{2}(p)$ in a series of $p$

$$
\begin{aligned}
y(\tau, p) & =y_{(k-1)}(\tau, p)+p^{k} y_{k}(\tau)+\cdots, \\
\omega^{2}(p) & =\omega_{(k-1)}^{2}(p)+p^{k} \omega_{k}+\cdots,
\end{aligned}
$$

in which

$$
\begin{aligned}
y_{(k-1)}(\tau, p) & =y_{0}(\tau)+p y_{1}(\tau)+\cdots+p^{k-1} y_{k-1}(\tau), \\
\omega_{(k-1)}^{2}(p) & =\omega_{0}^{2}+p \omega_{1}+\cdots+p^{k-1} \omega_{k-1} .
\end{aligned}
$$

Obviously, when $p=0, y(t, 0)$ is the zeroth-order approximation and when $p=1, y(t, 1)$ is the required approximate solution of (3). Generally, (9) provides the higher-order approximation to the exact solution. For example, the firstorder analytical approximation turns out to be

$$
\begin{aligned}
y(\tau) & =y_{0}(\tau)+p y_{1}(\tau), \\
\omega^{2} & =\omega_{0}^{2}+p \omega_{1} .
\end{aligned}
$$

Substituting (11) into (3) and equating the coefficients of the $p$ yield

$$
F_{1}\left(\tau, \omega_{1}, y_{1}(\tau)\right)=\omega_{0}^{2} y_{1}^{\prime \prime}+\omega_{1} y_{0}^{\prime \prime}-\frac{d f}{d y}\left(y_{0}\right) y_{1} .
$$

By considering (4) and harmonic balance method, let

$$
y_{1}(\tau)=a_{3,1}(\cos \tau-\cos 3 \tau)
$$

Substituting (13) into (12), we consider the following equation:

$$
R_{1}(\tau)=F_{1}\left(\tau, \omega_{1}, y_{1}(\tau)\right)+R_{0}(\tau)
$$

in this way, the initial residual $R_{0}(\tau)$ is introduced into (14) to improve the accuracy.

According to harmonic balance method, the right-hand side of (14) should not contain the terms $\cos \tau, \cos 3 \tau$. Letting their coefficients be zeros yields two linear equations with two unknowns $\omega_{1}$ and $a_{3,1}$, through which the two unknowns can be solved easily. We now get the first-order approximation

$$
\begin{aligned}
y_{(1)}(\tau) & =y_{0}(\tau)+y_{1}(\tau), \\
\omega_{(1)}^{2} & =\omega_{0}^{2}+\omega_{1}, \\
\tau & =\omega_{(1)} t
\end{aligned}
$$

with the residual

$$
R_{1}(\tau)=\omega_{(1)}^{2} y_{(1)}^{\prime \prime}-f\left(y_{(1)}\right),
$$

where $y_{0}(\tau)$ and $y_{1}(\tau)$ are given by (7) and (13), respectively. 
With the process going on, the higher-order approximation can be obtained similarly. For example, for the $k$ th-order approximation, we may assume

$$
y_{k}(\tau)=\sum_{i=1}^{k} a_{2 i+1, k}[\cos \tau-\cos (2 i+1) \tau],
$$

$$
k=2,3, \ldots
$$

To determine the unknown parameters $a_{2 i+1, k}(i=2$, $\ldots, k)$ and $\omega_{k}$, we substitute (9) into (3) and collect the coefficients of $p$ and finally we get

$$
\begin{aligned}
F_{k}\left(\tau, \omega_{k}, y_{k}(\tau)\right)= & \omega_{0}^{2} y_{k}^{\prime \prime}+\sum_{i=1}^{k} \omega_{i} y_{k-i}^{\prime \prime} \\
& +\left.\frac{1}{k !} \frac{d^{k}}{d p^{k}}(f(y))\right|_{p=0} .
\end{aligned}
$$

Substituting $y_{(k-1)}(\tau, 1)$ and $\omega_{(k-1)}^{2}(1)$ into (3), one yields the following residual:

$$
R_{k-1}(\tau)=\omega_{(k-1)}^{2}(1) y_{(k-1)}^{\prime \prime}(\tau, 1)-f\left(y_{(k-1)}(\tau, 1)\right) .
$$

Let us consider the equation

$$
R_{k}(\tau)=F_{k}\left(\tau, \omega_{k}, y_{k}(\tau)\right)+R_{k-1}(\tau)
$$

and eliminate the contributions proportional to $\cos \tau, \cos 3 \tau$, $\ldots$ and $\cos (2 k+1) \tau$ in the right-hand side of $(20)$; we can solve the unknown $a_{3, k}, a_{5, k}, \ldots, a_{2 k+1, k}$ and $\omega_{k}$ from those equations.

Then, the $k$-order approximations to the frequency and periodic solution can be obtained in the form

$$
\begin{gathered}
y_{(k)}(\tau)=y_{(k-1)}(\tau, 1)+y_{k}(\tau), \\
\omega_{(k)}^{2}=\omega_{(k-1)}^{2}(1)+\omega_{k},
\end{gathered}
$$

where $y_{(k-1)}(\tau, 1)$ and $\omega_{(k-1)}^{2}(1)$ are given by $(10)$.

\section{Solution Procedure}

By introducing a new time scale $\tau=\omega t$, then (1) becomes

$$
\begin{aligned}
\omega^{2} y^{\prime \prime}+\alpha y+\beta y^{3}+\gamma y^{1 / 3} & =0, \\
y(0) & =A, \\
y^{\prime}(0) & =0,
\end{aligned}
$$

where prime denotes the derivative with respect $\tau$ and $\omega$ is an angular frequency to be determined.

By following (7), the initial approximation with the initial conditions can be written into

$$
\begin{gathered}
y_{0}(\tau)=A \cos \tau, \\
\omega=\omega_{0} .
\end{gathered}
$$

Here, it is possible to do the following Fourier series expansion:

$$
(\cos \tau)^{1 / 3}=\sum_{n=0}^{\infty} a_{2 n+1} \cos (2 n+1) \tau
$$

in which

$$
\begin{aligned}
a_{2 n+1} & =\frac{4}{\pi} \int_{0}^{\pi / 2}(\cos \tau)^{1 / 3} \cos [(2 n+1) \tau] d \tau \\
& =\frac{3 \Gamma(7 / 3)}{2^{4 / 3} \Gamma(n+5 / 3) \Gamma(2 / 3-n)} .
\end{aligned}
$$

Substituting (23) and (24) into (22) yields

$$
R_{0}(\tau)=F_{01} \cos \tau+F_{03} \cos 3 \tau+F_{05} \cos 5 \tau+\cdots,
$$

in which

$$
\begin{aligned}
& F_{01}=\alpha A-\omega_{0}^{2} A+0.75 \beta A^{3}+1.1596 \gamma A^{1 / 3}, \\
& F_{03}=0.25 \beta A^{3}-0.231919 \gamma A^{1 / 3}, \\
& F_{05}=0.11596 \gamma A^{1 / 3} .
\end{aligned}
$$

Eliminating contributions proportional to $\cos \tau$ in (26), that is, solving equation $F_{01}=0$, gives

$$
\omega_{0}=\sqrt{\alpha+0.75 \beta A^{2}+1.1596 \gamma A^{-2 / 3}},
$$

which is the same as the one by $\mathrm{Xu}$ [24]. Till now we get the zeroth-order analytical approximation (23) and the initial residual

$$
R_{0}(\tau)=F_{03} \cos 3 \tau+F_{05} \cos 5 \tau+\cdots .
$$

To obtain the first-order analytical solution, we substitute (11) and (13) into (12), expand the function $(\cos \tau-$ $\cos 3 \tau)(\cos \tau)^{-2 / 3}$ into a Fourier series

$$
(\cos \tau-\cos 3 \tau)(\cos \tau)^{-2 / 3}=\sum_{n=0}^{\infty} b_{2 n+1} \cos (2 n+1) \tau,
$$

in which

$$
\begin{aligned}
& b_{2 n+1}=\frac{4}{\pi} \int_{0}^{\pi / 2}(\cos \tau-\cos 3 \tau)(\cos \tau)^{-2 / 3} \\
& \cdot \cos [(2 n+1) \tau] d \tau,
\end{aligned}
$$

and finally get

$$
F_{1}\left(\tau, \omega_{1}\right)=\frac{F_{11} \cos \tau+F_{13} \cos 3 \tau+F_{15} \cos 5 \tau}{16}+\cdots,
$$

in which

$$
\begin{aligned}
& F_{11}=0.75 a_{3,1} \beta A^{2}+\omega_{1} A-0.695757 a_{3,1} \gamma A^{-2 / 3}, \\
& F_{13}=a_{3,1}\left(8 \alpha+6 \beta A^{2}+9.85656 \gamma A^{-2 / 3}\right), \\
& F_{15}=-0.75 a_{3,1} \beta A^{2}+0.17921 a_{3,1} \gamma A^{-2 / 3} .
\end{aligned}
$$


According to (16), we have

$$
R_{1}(\tau)=F_{1}\left(\tau, \omega_{1}\right)+R_{0}(\tau)
$$

Setting the coefficients of $\cos \tau, \cos 3 \tau$ to zero in the righthand side of (34) gives

$$
\begin{aligned}
0= & 0.75 a_{3,1} \beta A^{2}+\omega_{1} A-0.695757 a_{3,1} \gamma A^{-2 / 3}, \\
0= & 0.25 \beta A^{3}-0.231919 \gamma A^{1 / 3} \\
& +a_{3,1}\left(8 \alpha+6 \beta A^{2}+9.85656 \gamma A^{-2 / 3}\right) .
\end{aligned}
$$

Solving (35) yields

$$
\begin{aligned}
& \omega_{1} \\
& =-\frac{0.00624971\left(12.0006 \beta A^{8 / 3}-11.1326 \gamma\right)^{2}}{A^{2 / 3}\left(38.4018 \alpha A^{2 / 3}+28.8013 \beta A^{8 / 3}+47.3137 \gamma\right)}, \\
& a_{3,1}=\frac{1.11326 \gamma A-1.20006 \beta A^{11 / 3}}{38.4018 \alpha A^{2 / 3}+28.8013 \beta A^{8 / 3}+47.3137 \gamma} .
\end{aligned}
$$

Thus, we obtain the first-order approximation

$$
\begin{aligned}
\omega_{(1)} & =\sqrt{\omega_{0}^{2}+\omega_{1}}, \\
y_{(1)}(t) & =\left(A+a_{3,1}\right) \cos \left(\omega_{(1)} t\right)-a_{3,1} \cos \left(3 \omega_{(1)} t\right),
\end{aligned}
$$

with the residual

$$
\begin{aligned}
R_{1}(\tau)= & 0.11596 \gamma A^{1 / 3}-0.75 a_{3,1} \beta A^{2} \\
& +0.17921 a_{3,1} \gamma A^{-2 / 3}+\cdots
\end{aligned}
$$

where $\omega_{0}, \omega_{1}$, and $a_{3,1}$ are determined by (28), (36), and (37), respectively.

With the procedure going on, similarly, we can get the high-order approximation. For example, the second-order approximation can be obtained as follows:

$$
\begin{aligned}
y_{(2)}(t)= & \left(A+a_{3,1}+a_{3,2}+a_{5,2}\right) \cos \omega_{(2)} t \\
& -\left(a_{3,1}+a_{3,2}\right) \cos 3 \omega_{(2)} t \\
& -a_{5,2} \cos 5 \omega_{(2)} t, \\
\omega_{(2)}= & \sqrt{\omega_{0}^{2}+\omega_{1}+\omega_{2}},
\end{aligned}
$$

in which

$$
\begin{aligned}
a_{3,2} & =\frac{G}{L^{2} M}, \\
a_{5,2} & =\frac{H}{L^{2} M}, \\
\omega_{2} & =\frac{F}{A^{2 / 3} L^{2} M} .
\end{aligned}
$$

The variables $L, M, G, H$, and $F$ are presented in Appendix.

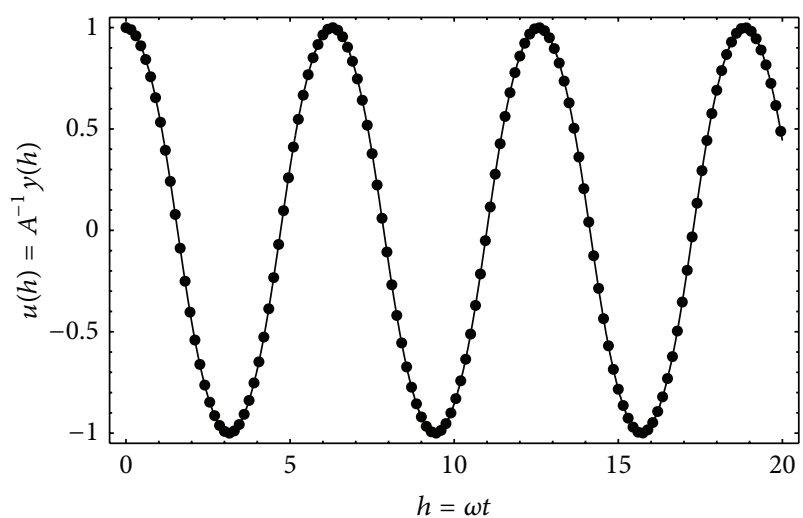

FIGURE 1: Comparison of the approximate solution with the exact solution; • • e exact solution, -2 nd-order approximate solution.

\section{Results and Discussion}

In order to illustrate and verify the efficiency and correctness of the presented approach for this strongly nonlinear oscillator, we consider some special cases.

Case 1. If $\alpha=0, \beta=0, \gamma=1$, (1) reduces to

$$
\begin{gathered}
\ddot{y}+y^{1 / 3}=0, \\
y(0)=A, \\
y^{\prime}(0)=0 .
\end{gathered}
$$

Then, from (41), the second-order approximation of the frequency can be obtained:

$$
\omega_{(2)}=\sqrt{1.1466 A^{-2 / 3}}=1.07079 A^{-1 / 3}
$$

The exact frequency [16] is $\omega=1.070451 A^{-1 / 3}$. Therefore, it can be easily proved that the maximal relative error is less than $0.032 \%$.

Hence, from (40), the second-order approximate solution can be expressed as follows:

$$
\begin{aligned}
y_{(2)}(t)= & 1.01966 A \cos \left(\omega_{(2)} t\right) \\
& -0.0238744 A \cos \left(3 \omega_{(2)} t\right) \\
& +0.00421451 A \cos \left(5 \omega_{(2)} t\right),
\end{aligned}
$$

which agrees very well with the exact solution [6] as shown in Figure 1.

To further illustrate and verify the accuracy of the presented approach in this case, we present the comparison between the approximate and exact frequencies for the second-order approximation by using different methods in Table 1. It is clear that, for the second-order approximation, the result obtained in this paper is better than those obtained previously by other authors. 

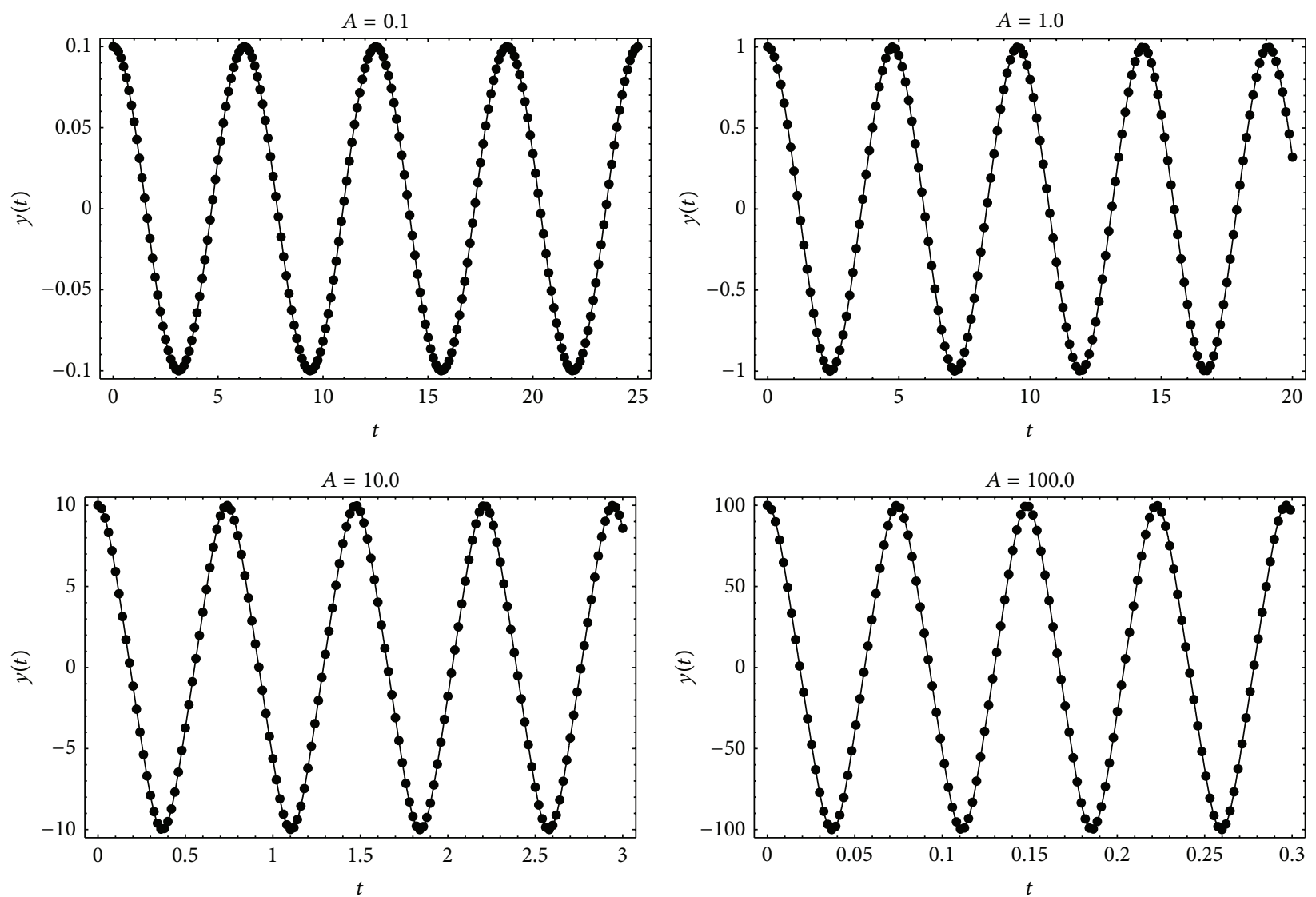

FIGURE 2: Comparison of the approximate solution with the exact solution; $\bullet-\cdots$ exact solution, — 2nd-order approximate solution.

TABLE 1: Comparison of the exact and approximate frequencies (2nd-order) obtained by using different methods.

\begin{tabular}{lccccc}
\hline & This paper & HPM [16] & HPM [17] & HBM [11] & HBM [12] \\
\hline$A^{1 / 3} \omega_{2}$ & 1.07079 & 1.06861 & 1.06991 & 1.06928 & 1.06341 \\
$\%$ error & $0.032 \%$ & $0.17 \%$ & $0.050 \%$ & $0.11 \%$ & $0.66 \%$ \\
\hline
\end{tabular}

Case 2. If $\alpha=1, \beta=\epsilon, \gamma=0$, (1) reduces to a Duffing equation

$$
\begin{aligned}
& \ddot{y}+y+\epsilon y^{3}=0 \text {, } \\
& y(0)=A, \\
& y^{\prime}(0)=0 .
\end{aligned}
$$

In this case, the first- and second-order approximate periods obtained by (38) and (41) for different values of $\epsilon A^{2}$ are shown in Table 2 . The relative errors (RE) are defined as $\mathrm{RE}=\left|\left(T-T_{\mathrm{ex}}\right) / T_{\mathrm{ex}}\right| \times 100$. It can be observed that the approximate periods have a good adjustment with the exact ones.

To verity results, Figure 2 shows the comparisons of the second-order analytical solutions obtained by (40) with the exact ones for $A=0.1,1,10$, and 100 when $\epsilon=1$. It can be seen from this figure that our analytical results are very close
TABLE 2: Comparison of approximate periods with the exact period for Case 2.

\begin{tabular}{lccc}
\hline$\epsilon A^{2}$ & $T_{(1)}(\mathrm{RE})$ & $T_{(2)}(\mathrm{RE})$ & $T_{\mathrm{ex}}$ \\
\hline 0.01 & $6.25976(0.00000)$ & $6.25976(0.00000)$ & 6.25976 \\
0.1 & $6.06066(0.00000)$ & $6.06066(0.00000)$ & 6.06066 \\
1 & $4.76792(0.00210)$ & $4.76803(0.00021)$ & 4.76802 \\
10 & $2.19094(0.03878)$ & $2.19191(0.00548)$ & 2.19179 \\
100 & $0.75814(2.97178)$ & $0.73633(0.00951)$ & 0.73626 \\
1000 & $0.234194(0.06230)$ & $0.23437(0.01281)$ & 0.23434 \\
\hline
\end{tabular}

to the exact ones for the wide range of initial amplitude in this case.

Case 3. If $\alpha=1, \beta=0, \gamma=1$, we can obtain the following nonlinear oscillator:

$$
\begin{array}{r}
\ddot{y}+y+y^{1 / 3}=0, \\
y(0)=A, \\
y^{\prime}(0)=0 .
\end{array}
$$

From Table 3 and Figure 3, we can see that the accuracy of the results obtained in this paper is in excellent agreement with exact ones for the wide range of initial amplitude $A$. 

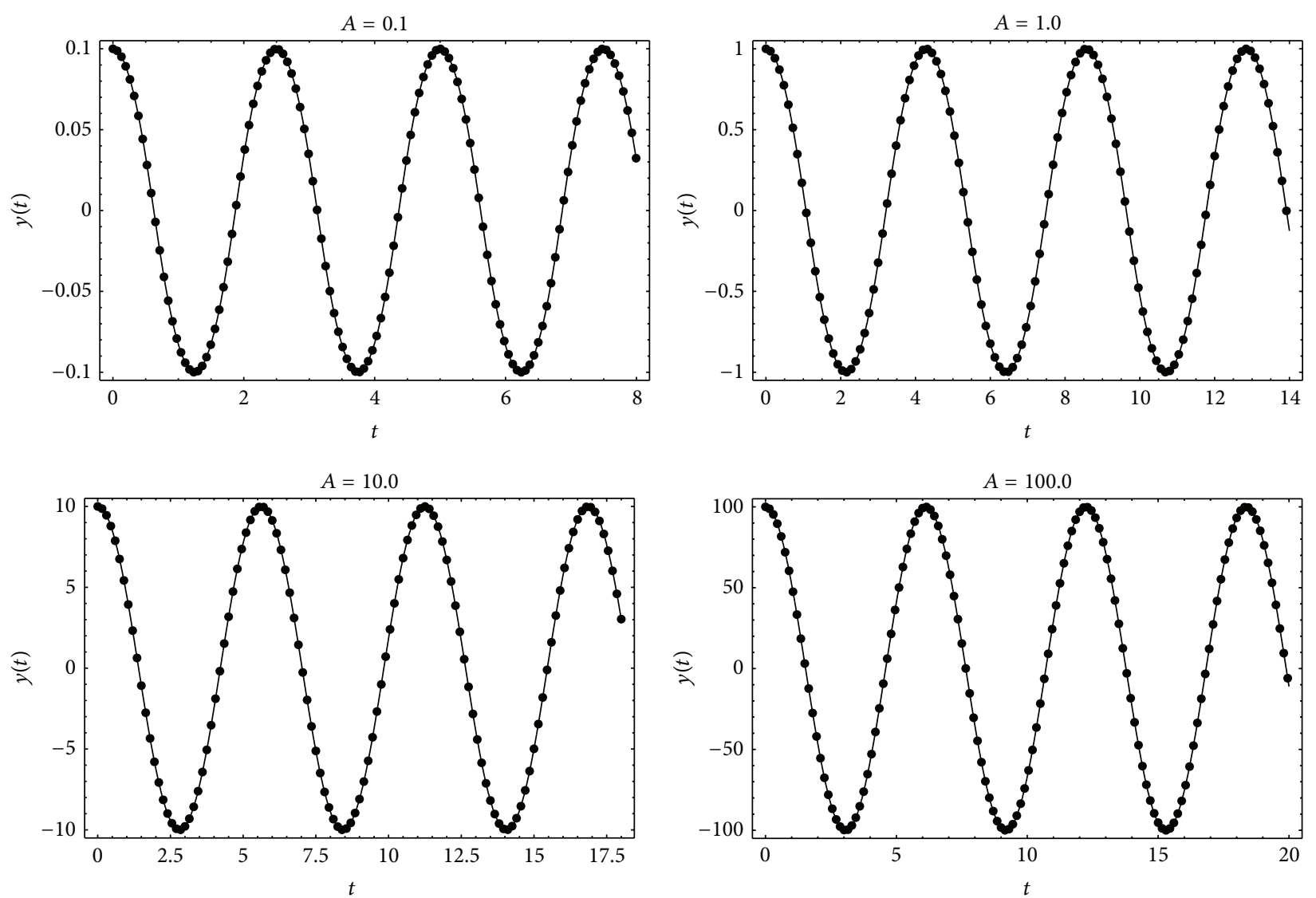

FIGURE 3: Comparison of the approximate solution with the exact solution; $\bullet-\cdots$ exact solution, - 2nd-order approximate solution.

TABLE 3: Comparison of approximate periods with the exact period for Case 3.

\begin{tabular}{lccc}
\hline$A^{2}$ & $T_{(1)}(\mathrm{RE})$ & $T_{(2)}(\mathrm{RE})$ & $T_{\mathrm{ex}}$ \\
\hline 0.01 & $1.24079(0.10731)$ & $1.23909(0.02985)$ & 1.23946 \\
0.1 & $2.49977(0.08246)$ & $2.49714(0.02282)$ & 2.49771 \\
1 & $4.28454(0.03385)$ & $4.28271(0.00887)$ & 4.28309 \\
10 & $5.62191(0.00481)$ & $5.62157(0.00125)$ & 5.62164 \\
100 & $6.12075(0.00033)$ & $6.12072(0.00016)$ & 6.12073 \\
1000 & $6.24708(0.00016)$ & $6.24707(0.00000)$ & 6.24707 \\
\hline
\end{tabular}

\section{Conclusions}

An iterative homotopy harmonic balance approach has been presented and applied to deduce the accurate approximations to the angular frequency and periodic solution of a strongly nonlinear oscillator. The high-order analytical approximations of the frequency and solution of this oscillator are obtained. Excellent agreement between approximate results by this approach and the exact ones has been demonstrated and discussed; the discrepancy between the second-order approximate results and exact ones is very very low. And, we can see that the approach considered here is very simple in its principle and has great potential to be applied to other nonlinear oscillators.

\section{Appendix}

Consider

$$
\begin{aligned}
L= & 38.402 \alpha A^{2 / 3}+28.801 \beta A^{8 / 3}+47.314 \gamma \\
M= & 8.9220 \times 10^{6} \alpha^{2} A^{4 / 3}+1.3104 \times 10^{7} \alpha \beta A^{10 / 3} \\
& +4.8095 \times 10^{6} \beta^{2} A^{16 / 3}+2.1581 \\
& \times 10^{7} \alpha \gamma A^{2 / 3}+1.5845 \times 10^{7} \beta \gamma A^{8 / 3} \\
& +1.3046 \times 10^{7} \gamma^{2} \\
G= & 1.0434 \times 10^{7} \beta^{2} \gamma^{2} A^{19 / 3}+9.0854 \times 10^{6} \alpha \gamma^{3} A^{5 / 3} \\
& -2.0979 \times 10^{7} \beta \gamma^{3} A^{11 / 3}-7.2274 \\
& \times 10^{6} \alpha \beta^{3} A^{29 / 3}-5.1947 \times 10^{6} \beta^{4} A^{35 / 3} \\
& +7.6682 \times 10^{6} \beta^{3} \gamma A^{9}-1.3681 \\
& \times 10^{8} \alpha \beta \gamma^{3} A^{10 / 3}-2.2453 \times 10^{7} \alpha \beta \gamma^{2} A^{13 / 3} \\
& +502610 \alpha^{2} \gamma^{2} A^{7 / 3}+1.0075 \times 10^{7} \gamma^{4} A \\
& +2.2603 \times 10^{7} \alpha \beta^{2} \gamma A^{7},
\end{aligned}
$$




$$
\begin{aligned}
& H=-1.8872 \times 10^{7} \alpha \beta^{3} A^{29 / 3}-7.1521 \times 10^{6} \beta^{4} A^{35 / 3} \\
&-6.3571 \times 10^{7} \alpha^{3} \gamma A^{3}-2.3782 \\
& \times 10^{8} \alpha^{2} \gamma^{2} A^{7 / 3}-4.1300 \times 10^{7} \beta^{3} \gamma A^{9} \\
&-1.2715 \times 10^{8} \beta^{2} \gamma^{2} A^{19 / 3}-1.2804 \\
& \times 10^{8} \alpha^{2} \beta \gamma A^{5}-1.2849 \times 10^{7} \alpha^{2} \beta^{2} A^{23 / 3} \\
&-3.1979 \times 10^{8} \alpha \beta \gamma^{2} A^{13 / 3}-1.1701 \\
& \times 10^{8} \alpha \beta^{2} \gamma A^{7}-2.9636 \times 10^{8} \alpha \gamma^{3} A^{5 / 3} \\
&-1.2308 \times 10^{8} \gamma^{4} A-1.9910 \\
& \times 10^{8} \beta \gamma^{3} A^{11 / 3}, \\
& F= .8520 \times 10^{7} \gamma^{5}-5.4206 \times 10^{6} \alpha \beta^{4} A^{34 / 3} \\
&-4.2348 \times 10^{6} \beta^{5} A^{40 / 3}-9.5356 \\
& \times 10^{7} \alpha^{3} \beta \gamma A^{14 / 3}-1.3755 \times 10^{8} \alpha \beta^{3} \gamma A^{26 / 3} \\
&+2.4354 \times 10^{8} \alpha \gamma^{4} A^{2 / 3}-1.3681 \\
& \times 10^{8} \alpha \beta \gamma^{3} A^{10 / 3}-2.4412 \times 10^{8} \alpha^{2} \beta \gamma^{2} A^{4} \\
&+1.8102 \times 10^{7} \beta \gamma^{4} A^{8 / 3}-1.7763 \\
& \times 10^{8} \beta^{3} \gamma^{2} A^{8}+1.9654 \times 10^{8} \alpha^{2} \gamma^{3} A^{4 / 3} \\
&+5.1601 \times 10^{7} \alpha^{3} \gamma^{2} A^{2}-2.4738 \\
& \times 10^{8} \beta^{2} \gamma^{3} A^{16 / 3}-4.8078 \times 10^{8} \alpha \beta^{2} \gamma^{2} A^{6} \\
&-2.9418 \times 10^{7} \beta^{4} \gamma A^{32 / 3}-2.1293 \\
& \times 10^{8} \alpha^{2} \beta^{2} \gamma A^{20 / 3} . \\
& \hline \\
& \hline
\end{aligned}
$$

\section{Conflict of Interests}

The authors declare that there is no conflict of interests regarding the publication of this paper.

\section{Acknowledgment}

The second author is supported by the National Science Foundation of China (11471118).

\section{References}

[1] V. Marinca and N. Herişanu, "Periodic solutions of Duffing equation with strong non-linearity," Chaos, Solitons \& Fractals, vol. 37, no. 1, pp. 144-149, 2008.

[2] A. A. Hemeda, "Variational iteration method for solving nonlinear partial differential equations," Chaos, Solitons and Fractals, vol. 39, no. 3, pp. 1297-1303, 2009.
[3] G. Wu, D. Baleanu, and Z. Deng, "Variational iteration method as a kernel constructive technique," Applied Mathematical Modelling, vol. 39, no. 15, pp. 4378-4384, 2015.

[4] A. A. Hemeda, "Variational iteration method for solving wave equation," Computers and Mathematics with Applications, vol. 56, no. 8, pp. 1948-1953, 2008.

[5] L. Xu, "He's parameter-expanding methods for strongly nonlinear oscillators," Journal of Computational and Applied Mathematics, vol. 207, no. 1, pp. 148-154, 2007.

[6] J.-H. He, "Some asymptotic methods for strongly nonlinear equations," International Journal of Modern Physics B, vol. 20, no. 10, pp. 1141-1199, 2006.

[7] M. T. Darvishi, A. Karami, and C. S. Byeong, "Application of He's parameter-expansion method for oscillators with smooth odd nonlinearities," Physics Letters, Section A: General, Atomic and Solid State Physics, vol. 372, no. 33, pp. 5381-5384, 2008.

[8] T. Detroux, L. Renson, L. Masset, and G. Kerschen, "The harmonic balance method for bifurcation analysis of large-scale nonlinear mechanical systems," Computer Methods in Applied Mechanics and Engineering, vol. 296, pp. 18-38, 2015.

[9] M. A. Hosen and M. S. H. Chowdhury, "A new analytical technique based on harmonic balance method to determine approximate periods for Duffing-harmonic oscillator," Alexandria Engineering Journal, vol. 54, no. 2, pp. 233-239, 2015.

[10] H. Hu and J. H. Tang, "Solution of a Duffing-harmonic oscillator by the method of harmonic balance," Journal of Sound and Vibration, vol. 294, no. 3, pp. 637-639, 2006.

[11] C. W. Lim and B. S. Wu, "Accurate higher-order approximations to frequencies of nonlinear oscillators with fractional powers," Journal of Sound and Vibration, vol. 281, no. 3-5, pp. 1157-1162, 2005.

[12] R. E. Mickens, "Oscillations in an $x 4 / 3$ potential," Journal of Sound and Vibration, vol. 246, no. 2, pp. 375-378, 2001.

[13] Y.-S. Yun and C. Temuer, "Application of the homotopy perturbation method for the large deflection problem of a circular plate," Applied Mathematical Modelling, vol. 39, no. 3-4, pp. 1308-1316, 2015.

[14] H. S. Najafi and S. A. Edalatpanah, "Homotopy perturbation method for linear programming problems," Applied Mathematical Modelling, vol. 38, no. 5-6, pp. 1607-1611, 2014.

[15] A. A. Hemeda, "Homotopy perturbation method for solving systems of nonlinear coupled equations," Applied Mathematical Sciences, vol. 6, no. 93-96, pp. 4787-4800, 2012.

[16] A. Beléndez, C. Pascual, S. Gallego, M. Ortuño, and C. Neipp, "Application of a modified He's homotopy perturbation method to obtain higher-order approximations of an $x^{1 / 3}$ force nonlinear oscillator," Physics Letters A, vol. 371, no. 5-6, pp. 421-426, 2007.

[17] A. Beléndez, "Homotopy perturbation method for a conservative $x^{1 / 3}$ force nonlinear oscillator," Computers and Mathematics with Applications, vol. 58, pp. 2267-2273, 2009.

[18] T. Öziş and A. Yildirim, "Determination of the frequencyamplitude relation for a Duffing-harmonic oscillator by the energy balance method," Computers and Mathematics with Applications, vol. 54, no. 7-8, pp. 1184-1187, 2007.

[19] S. S. Ganji, D. D. Ganji, Z. Z. Ganji, and S. Karimpour, "Periodic solution for strongly nonlinear vibration systems by He's Energy balance method," Acta Applicandae Mathematicae, vol. 106, no. 1, pp. 79-92, 2009.

[20] J.-H. He, "A note on delta-perturbation expansion method," Applied Mathematics and Mechanics, vol. 23, no. 6, pp. 634-638, 2002. 
[21] Z. Guo, A. Y. T. Leung, and H. X. Yang, "Iterative homotopy harmonic balancing approach for conservative oscillator with strong odd-nonlinearity," Applied Mathematical Modelling, vol. 35, no. 4, pp. 1717-1728, 2011.

[22] Z. Guo and A. Y. T. Leung, "The iterative homotopy harmonic balance method for conservative Helmholtz-Duffing oscillators," Applied Mathematics and Computation, vol. 215, no. 9, pp. 3163-3169, 2010.

[23] A. Y. T. Leung and Z. Guo, "Residue harmonic balance approach to limit cycles of non-linear jerk equations," International Journal of Non-Linear Mechanics, vol. 46, no. 6, pp. 898-906, 2011.

[24] L. Xu, "Determination of limit cycle by He's parameter-expanding method for strongly nonlinear oscillators," Journal of Sound and Vibration, vol. 302, no. 1-2, pp. 178-184, 2007. 


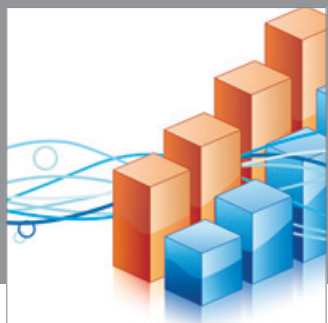

Advances in

Operations Research

mansans

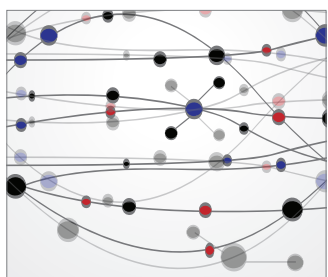

The Scientific World Journal
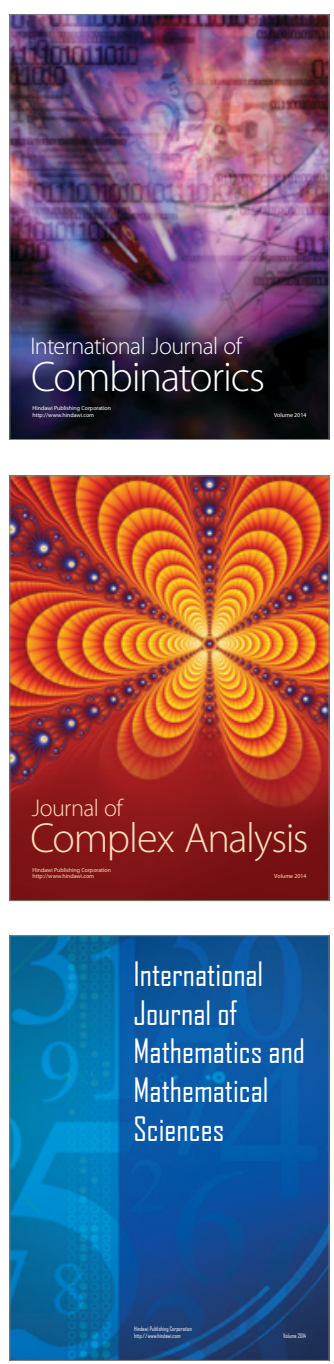
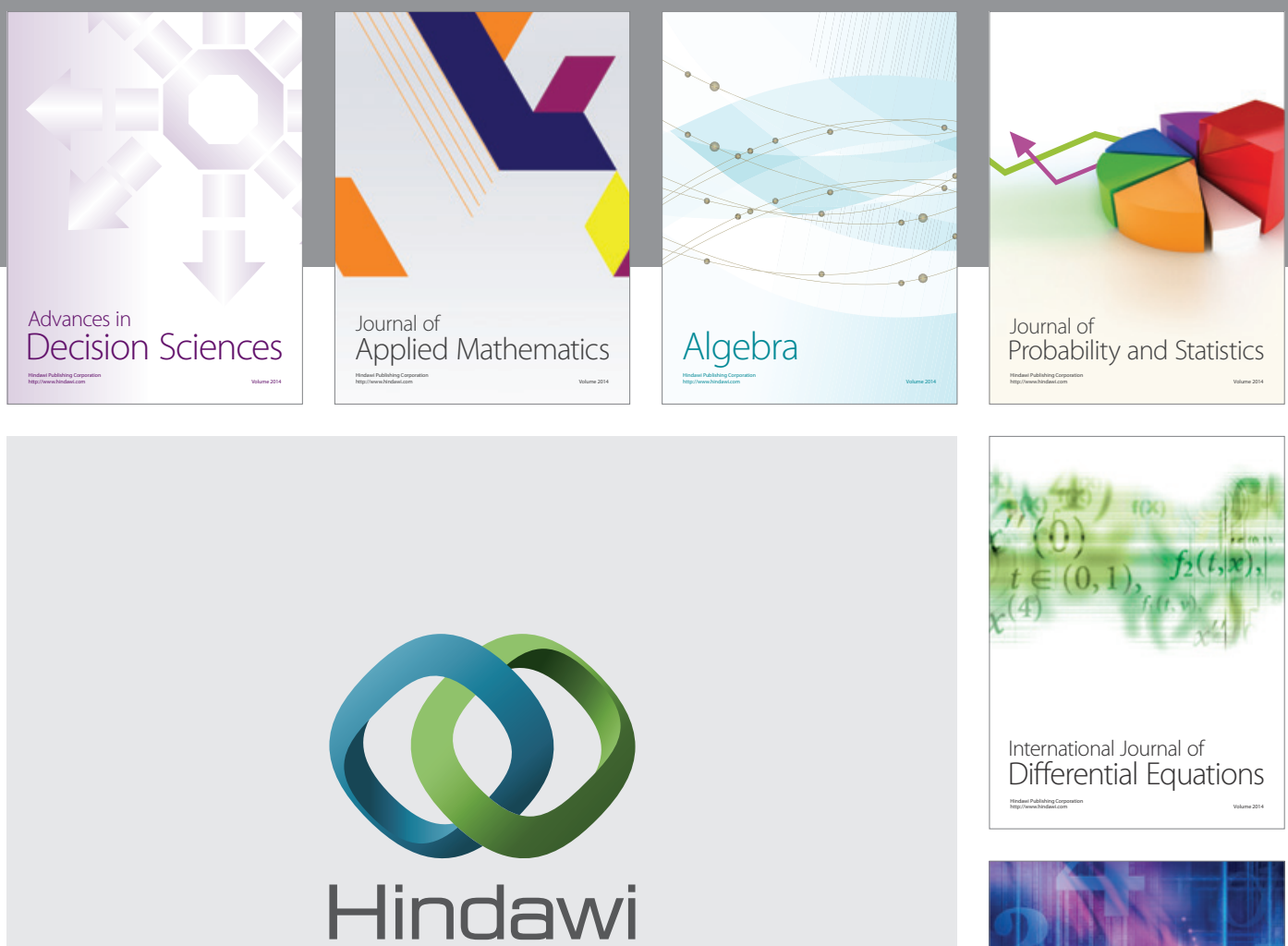

Submit your manuscripts at http://www.hindawi.com
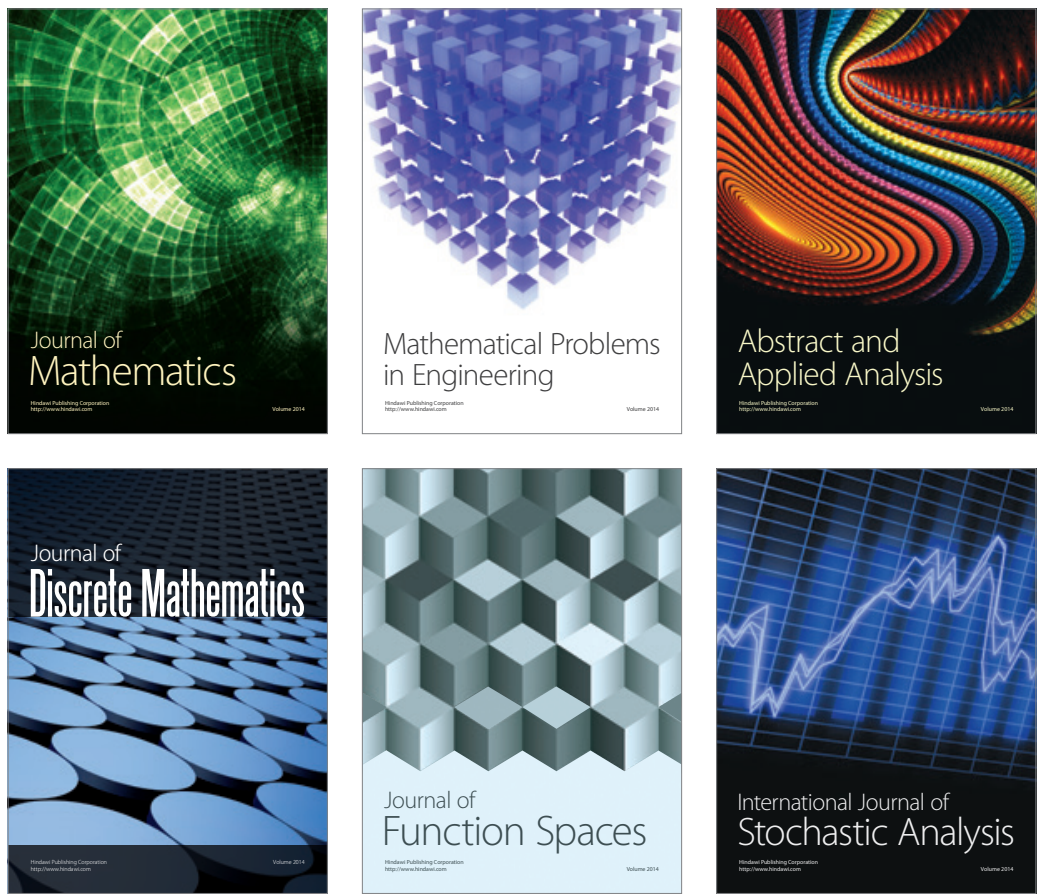

Journal of

Function Spaces

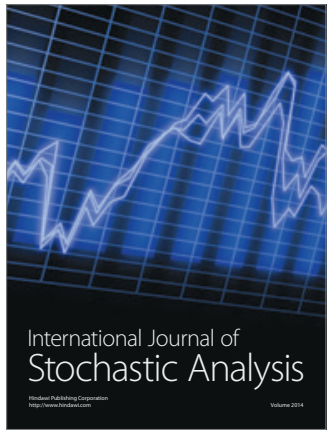

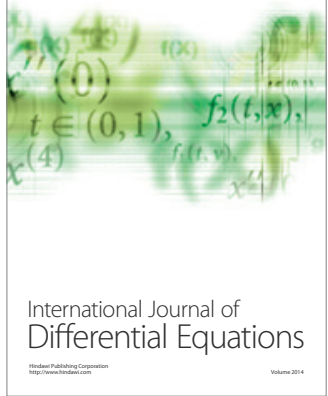
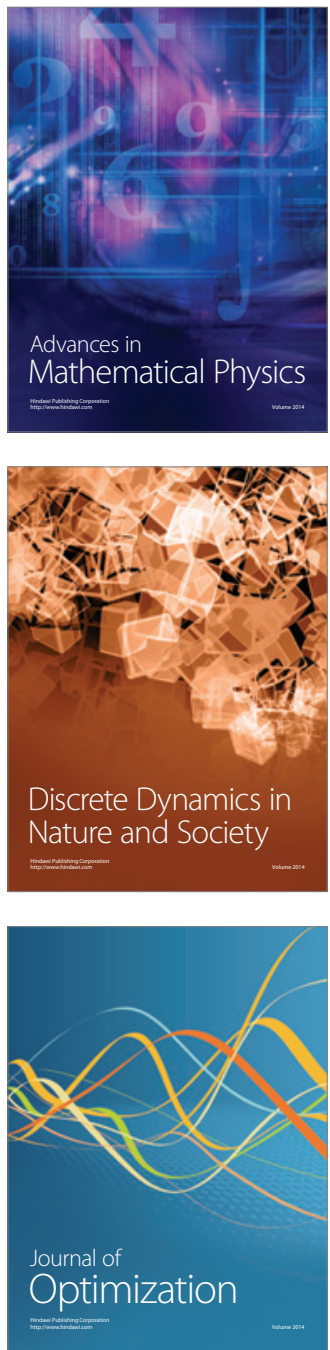